\title{
Mean flow structure of non-equilibrium boundary layers with adverse pressure gradient
}

\author{
B C MANDAL ${ }^{1, *}$, H P MAZUMDAR ${ }^{2}$ and S S DUTTA ${ }^{3}$ \\ ${ }^{1}$ Jalpaiguri Government Engineering College, Civil Engineering Department, \\ Jalpaiguri 735 102, India \\ ${ }^{2}$ Indian Statistical Institute, Physics and Applied Mathematics Unit, \\ Kolkata 700 108, India \\ ${ }^{3}$ River Research Institute, Salt Lake City, Kolkata 700 091, India \\ e-mail: bcmjgec@gmail.com; bcm_rri@yahoo.co.in
}

MS received 19 March 2013; revised 28 October 2013; accepted 28 February 2014

\begin{abstract}
In this paper Spalding's formulation for the law of the wall with constants modified by Persen is used to describe the inner region (viscous sub-layer and certain portion of logarithmic layer) and a wake law due to Persen is used to describe the wake region (outer region). These two laws are examined in the light of measured data by Marušić and Perry for non-equilibrium adverse pressure gradient layers. It is observed that structure of turbulence for this flow is well-described by these two laws. From the known structure of turbulence eddy viscosity for the flow under consideration is calculated. Self similarity in eddy viscosity is observed in the wall region.
\end{abstract}

Keywords. Non-equilibrium turbulent boundary layer; adverse pressure gradient; law of the wall; law of the wake.

\section{Introduction}

Clauser (1954a, b) initiated the study of the properties of equilibrium turbulent boundary layers by introducing an important parameter e.g.,

$$
\beta=\frac{\delta^{*}}{\tau_{w}} \frac{d p}{d x} .
$$

In Eq. (1), the pressure gradient is scaled by wall shear stress $\tau_{w}$ and the displacement thickness $\delta^{*}$. For equilibrium turbulent boundary layers $\beta$ is constant. Admittedly, these types of flows are difficult to generate and maintain under equilibrium condition (Castillo \& Wang 2004). All such equilibrium flows with adverse pressure gradient, as presented in 1968 Stanford Conference were rated to be 'most difficult' and the methods followed for their treatment could not yield any accurate predictions.

\footnotetext{
*For correspondence
} 
Townsend (1976) developed an attached eddy hypothesis for zero pressure gradient boundary layers. His theory appeared to be more rigorous than that of Clauser (1954a, b), but the problem is reduced to an over constrained system when the velocity scale is used for its description. Such hypothesis was extended by Perry \& Marušić (1995) to boundary layer with stream-wise pressure gradient for both favourable and adverse cases. In a subsequent paper, Marušić \& Perry (1995) applied the formulation to non-equilibrium layers data measured by them and found good agreement with the theory. In this work Marušić and Perry also showed that wake parameter, $\Pi$ due to Coles (1956) remains, approximately invariant with the stream-wise distance for equilibrium turbulent boundary layer. They however, noticed that such condition breaks down for the case of non-equilibrium layers because the stream-wise derivative of Coles wake parameter starts playing significant role in momentum balance. Castillo \& George (2001) found that the proper velocity scale for the outer part of the boundary layer to be a free stream velocity in place of friction velocity. According to them, an equilibrium boundary layer might exist if the pressure parameter, given by

is a constant.

$$
\Lambda=\frac{\delta}{\rho U_{o}^{2} d \delta / d x} \frac{d p}{d x}
$$

Gorin \& Sikovsky (1998) carried out an asymptotic analysis of turbulent boundary layers with strong adverse pressure gradient. They derived limiting laws for velocity and turbulent stresses in the corresponding regions of the boundary layer. The turbulent boundary layer is shown to consist of three regions e.g., the constant stress layer, linear stress layer and the wake region.

Kornilov \& Litvinenko (2001) measured velocity profiles and applied various techniques for measuring coefficients of skin friction in incompressible turbulent boundary layer over a flat plate under conditions of a stream-wise adverse pressure gradients.

Materny et al (2008) have reported the results of their careful measurements on the influence of adverse pressure gradient on the turbulent boundary layer at the flat plate for large Reynolds number. They showed highly interesting result that such turbulent boundary layer at adverse pressure gradient conditions can be described by two velocity scales e.g., the inner (imposed by inner boundary condition from constant shear stress layer) and outer (imposed by outer layer) velocity scales.

In the present paper, we shall examine the applicability of two velocity distribution laws, (i) Spalding's (1961) law with constants modified by Persen (1976) and (ii) wake law due to Persen (1976), to the non-equilibrium turbulent boundary layer flow.

\section{Concept on two layered structure}

In many models of turbulent boundary layers, the structure of layer were thought of as consisting of three regions, e.g., (I) an inner region very close to the wall where viscous shear dominates, (III) an outer region where turbulent shear dominates and in between, (II) an overlap region where both types of shear are important. This concept may be changed with the introduction of Spalding's (1961) law of the wall. According to Persen (1976), the turbulent boundary layer is thought of as consisting of two regions:

(a) Region I (inner region), where Spalding's law of the wall as modified by Persen (1976) governs the flow and this region is unaffected by outside manipulation (pressure changes, history, etc.)

(b) Region II (outer region), where law of the wake as formulated by Persen (1976) governs the flow and this region is affected by outside manipulation. 
One basic idea follows from dimensional consideration is that the law of the wall may be expressed in the form:

$$
u^{+}=f\left(y^{+}\right),
$$

where $u^{+}=u / v_{*}$ and $y^{+}=y v_{*} / v ; u$ is the stream-wise mean velocity; $y$ is the distance from the wall; $v_{*}$ is the friction velocity, defined by $v_{*}^{2}=\tau_{w} / \rho, \tau_{w}$ being the wall shear stress; $\rho$ and $v$ are the density and kinematic viscosity of the fluid.

Spalding (1961) formulated the law of the wall as given below

$$
y^{+}=u^{+}+A\left[\exp \left(\kappa u^{+}\right)-1-\kappa u^{+}-\frac{\left(\kappa u^{+}\right)^{2}}{2}-\frac{\left(\kappa u^{+}\right)^{3}}{6}-\frac{\left(\kappa u^{+}\right)^{4}}{24}\right] .
$$

The formulation (4) has the advantage that it can be applied right from the wall. But Spalding's attempt to make the expression valid for the whole boundary layer with the choice of the constants $\kappa$ and $A$, respectively, as $\kappa=0.4$ and $A=0.1108$ was too ambitious.

Persen (1976) examined thoroughly the applicability of Spalding's formulation (4) against the voluminous data placed in the Stanford Conference (Coles \& Hirst 1969) on turbulent boundary layer and found the appropriate values of $\kappa$ and $A$, respectively, as

$$
\kappa=0.53227 \text { and } A=0.015 \text {, }
$$

which makes the Spalding formulation valid in the inner region.

The region II is the wake region wherein Coles' (1956) formulation is replaced by Persen (1976), which allows smooth matching of velocity profile at the upper edge of the wake region with the free stream velocity. Persen (1976) replaced the Coles' wake function with the expression

$$
\left(\frac{u^{+}-u_{\infty}^{+}}{\xi-u_{\infty}^{+}}\right)=\exp \left[\frac{-\left(y_{o}^{+}-y^{+}\right)^{2}}{\alpha^{2}}\right]
$$

where

$$
u \rightarrow U_{o} \text { as } \mathrm{y} \rightarrow \delta \text { and } u^{+} \rightarrow \frac{U_{o}}{v_{*}}=\xi \text { as } y^{+} \rightarrow \frac{\delta v_{*}}{v}=y_{o}^{+}
$$

$u_{\infty}^{+}=$constant, $U_{o}$ is the outer velocity (free stream velocity) and

$$
\frac{1}{\alpha^{2}}=\frac{\ln \left(\xi-u_{\infty}^{+}\right)-\ln \left(u_{1}^{+}-u_{\infty}\right)}{\left(y_{o}^{+}-y_{1}^{+}\right)^{2}}
$$

Here $\left(u_{1}^{+}, y_{1}^{+}\right)$is the point where law of the wall meets with the law of the wake.

The boundary layer ends up at $y^{+}=y_{o}^{+}$for which $u^{+}=\xi$. It is to be mentioned that the Spalding's formulation for the law of the wall (4) with values of constants, $A$ and $\kappa$ determined by Persen (1976) has an undisputed advantage that it satisfies no-slip condition and the proposed wake law exhibits a horizontal tangent at the outer edge $\left(u^{+}=\xi, y^{+}=y_{o}^{+}\right)$of the boundary layer. Further, the joining point of the 'near to wall region' with the 'wake region' is mathematically well-defined.

Mazumdar \& Mandal (2009) have shown that two layered concept in association with Eqs. (4), (5), and (6) is in good agreement with the turbulent boundary layer measurements of Österlund (1999).

Our objective is to examine that whether the same concept is applicable to the two-dimensional non-equilibrium boundary layer with adverse pressure gradient. 
In the present investigation, experimental data given by Marušić \& Perry (1995) for nonequilibrium adverse pressure gradient layers are brought into picture.

\section{Marušić and Perry's measurements: Adverse pressure gradient (APG)}

The experiments have been duly exposed to the profession through the published papers (Marušić \& Perry 1995; Perry \& Marušić 1995). For the sake of completeness of the paper a brief description of the experimental set-up is now presented.

The wind tunnel used in the experiment was of an open return blower type. The free-stream turbulence intensity was of the order of about $0.3 \%$. Wall was smooth and which was fitted with 68 pressure tapings spaced along its stream-wise centerline. The pressure gradient imposed along the smooth wall was established by heavily screening the rear of the diffuser section and by varying the angles of 12 adjustable louvers, which made up part of the roof of the working section. Initially, the flow is developed in a zero pressure gradient condition and then it is subjected to an approximately constant adverse pressure gradient. All turbulence measurements were made with $x$-wires. Mean flow profiles were measured using a pitot-static probe. Development of different boundary layer parameters are given in table 1 .

Marušić \& Perry (1995) determined shear velocity, $v_{*}$ from Clauser chart. Inspection of table 1 indicates that $v_{*}$ is a decaying function of downstream distance.

\subsection{Pressure gradient identification}

Coefficient of pressure $C_{p}$ is generally used to indicate strength of pressure gradient. A reference velocity obtained using an additional pitot-static tube, located at the beginning of the working section is used to define a coefficient of pressure $C_{p}$ which has the form

$$
C_{p}=\frac{P_{\text {local }}-P_{r e f}}{\frac{1}{2} \rho U_{r e f}^{2}}=1-\left(\frac{U_{o}}{U_{r e f}}\right)^{2},
$$

where $\rho$ fluid density, $P_{\text {local }}$ is local static pressure. $U_{o}$ is local free stream velocity; $P_{\text {ref }}$ and $U_{r e f}$ are the reference pressure and reference velocity respectively. Figure 1 shows the variation

Table 1. Development of boundary layers parameters.

\begin{tabular}{lcccccccccc}
\hline Flow & $\mathrm{x}, \mathrm{m}$ & $\mathrm{U}_{o}, \mathrm{~m} / \mathrm{s}$ & $\xi$ & $\nu^{*}, \mathrm{~m} / \mathrm{s}$ & $\delta, \mathrm{m}$ & $\delta^{*}, \mathrm{~m}$ & $\beta$ & $\beta$ (comp.) & $\theta, \mathrm{m}$ & $\operatorname{Re}_{\theta}$ \\
\hline 10 APG & 1.20 & 10.38 & 23.56 & 0.44 & 0.036 & 0.004 & $\sim 0.00$ & & 0.003 & 2068 \\
& 1.80 & 10.00 & 25.42 & 0.39 & 0.047 & 0.006 & 0.65 & 0.58 & 0.005 & 3023 \\
& 2.24 & 9.28 & 28.06 & 0.33 & 0.057 & 0.009 & 1.45 & 1.22 & 0.007 & 4056 \\
& 2.64 & 8.61 & 31.54 & 0.27 & 0.071 & 0.014 & 2.90 & 2.77 & 0.009 & 5312 \\
& 2.88 & 8.17 & 34.50 & 0.24 & 0.083 & 0.019 & 4.48 & 4.98 & 0.012 & 6282 \\
& 3.08 & 7.91 & 38.20 & 0.21 & 0.093 & 0.024 & 7.16 & 8.34 & 0.014 & 7190 \\
30 APG & 1.20 & 30.77 & 26.39 & 1.17 & 0.038 & 0.004 & $\sim 0.00$ & & 0.003 & 6027 \\
& 1.80 & 29.11 & 28.23 & 1.03 & 0.046 & 0.006 & 0.71 & 0.71 & 0.004 & 8230 \\
& 2.24 & 27.09 & 30.11 & 0.90 & 0.057 & 0.008 & 1.39 & 1.24 & 0.006 & 10685 \\
& 2.64 & 25.20 & 32.88 & 0.77 & 0.071 & 0.013 & 2.74 & 2.78 & 0.009 & 13940 \\
& 2.88 & 23.93 & 35.15 & 0.68 & 0.081 & 0.016 & 3.96 & 4.33 & 0.011 & 16347 \\
& 3.08 & 22.95 & 38.07 & 0.60 & 0.092 & 0.020 & 6.07 & 6.92 & 0.013 & 18890 \\
\hline
\end{tabular}




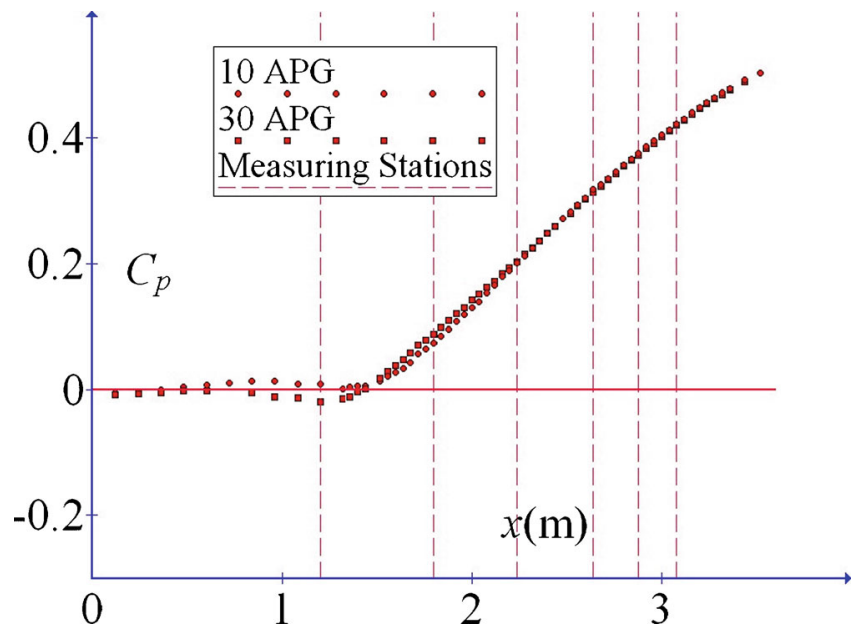

Figure 1. Values of coefficient of pressure plotted against downstream distance.

of $C_{p}$ along downstream distance $x$. The pressure distribution confirms that the flows encounter zero pressure gradient first, before its entering into constant adverse pressure gradient. The cases of flows with upstream velocity $U_{\text {ref }}$ set nominally to $10 \mathrm{~m} / \mathrm{s}$ (termed as 10 APG flow) and $30 \mathrm{~m} / \mathrm{s}$ (termed as 30 APG flow) are investigated.

The most interesting manipulation of the boundary layer is the one where it is subjected to the adverse pressure gradient. Eventually, it is necessary to identify the pressure gradient mathematically instead of stating it merely as 'strong', 'weak' or 'moderate'.

Figure 2 shows the variations of outside velocity $U_{o}$ for two flows (10 and 30 APG). Relation between $U_{o}$ and $x$ for 10 and $30 \mathrm{APG}$ case can be approximated as $U_{o}=$ $(-29.57 x+152.60)^{1 / 2}$ and $U_{o}=(-251.16 x+1298.13)^{1 / 2}$, respectively.

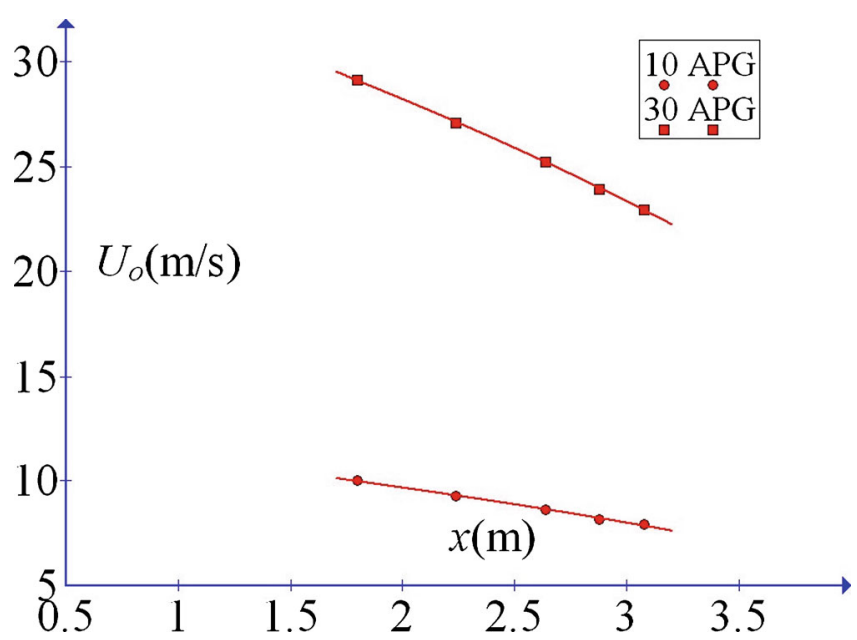

Figure 2. Free stream velocity as a function of downstream distance. 
The parameter $\beta$ can be rewritten as $\beta=\frac{\delta^{*}}{\tau_{w}}\left(-\rho U_{o} * d U_{o} / d x\right)$ and substituting $d U_{o} / d x$ from the above expressions, $\beta$ can be computed. The values of $\beta$ obtained in this way are given in $9^{\text {th }}$ column of table 1 , which are close to the values obtained by Marušić \& Perry (1995).

\subsection{Velocity profiles}

The developments of the stream-wise mean velocity profiles are shown in figure 3 for 10 APG flow. The velocity ' $u$ ' is normalized by the free stream velocity ' $U_{o}$ ' and wall distance ' $y$ ' is normalized by the boundary layer thickness ' $\delta$ '. Inspection of the velocity profiles indicate that the turbulent flow considered is under the influence of adverse pressure gradient.

Coles' defect profiles are plotted in figure 4 for 30 APG flow. It can be seen easily that the Coles' wake parameter $\Pi$ increases with the stream-wise distance, which indicates that the flow under consideration is in non-equilibrium state.

Mean deficit profiles are plotted in figure 5 in the coordinates proposed by Zagarola \& Smits (1998) for 10 APG flow. The figure shows that profiles do not collapse to a single curve (same result is obtained for 30 APG flow which is not shown here). This result is in qualitative agreement with that of Aubertine \& Eaton (2005). Reason may be attributed to the fact that at large Reynolds numbers, Zagarola and Smits' velocity scale becomes equivalent to the friction velocity (Maciel et al 2006).

We now turn to draw our attention to fit the velocity profiles of Marušić \& Perry (1995) with the Eqs. (4) and (6). The Eq. (4) is valid for $u^{+} \leq u_{1}^{+}$while, Eq. (6) is valid for $u_{1}^{+} \leq u^{+} \leq \xi$. Since $\left(u_{1}^{+}, y_{1}^{+}\right)$is a common point of the two curves, representing the law of the wall and the law of the wake, the following relation holds

$$
u_{1}^{+}=f\left(y_{1}^{+}\right) \text {. }
$$

The two curves must have a common tangent at that point. This tangent is given by $\tan \gamma$, where

$$
\tan \gamma=d u^{+} / d\left(\ln \left(y^{+}\right)\right)=y^{+} d u^{+} / d y^{+}
$$

here $\gamma$ is slope of velocity profile.



Figure 3. Mean flow development for 10 APG. 




Figure 4. Velocity defect profiles plot for 30 APG.

From Eq. (3) tangent

$$
\tan \gamma=y^{+} f^{\prime}\left(y^{+}\right)
$$

and, from Eq. (6), it follows that

$$
\tan \gamma=\frac{2}{\alpha^{2}}\left(u^{+}-u_{\infty}^{+}\right)\left(y_{o}^{+}-y^{+}\right) y^{+} .
$$

These expressions for $\tan \gamma$ must be equal at the point: $\left(u_{1}^{+}=u_{1}^{+}, y^{+}=y_{1}^{+}\right)$: Thus,

$$
y_{1}^{+} f^{\prime}\left(y_{1}^{+}\right)=\frac{2}{\alpha^{2}}\left(u_{1}^{+}-u_{\infty}^{+}\right)\left(y_{o}^{+}-y_{1}^{+}\right) y_{1}^{+} \text {. }
$$

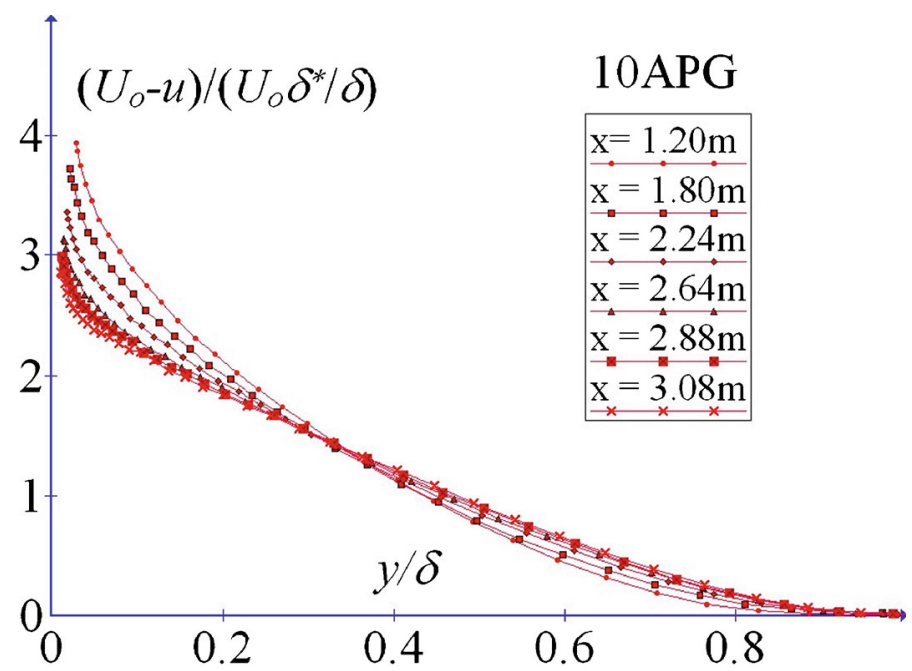

Figure 5. Velocity profiles plotted in Zagarola and Smits scaling. 
Once the values of $\xi$ and $y_{o}^{+}$are known, $u_{1}^{+}$and $y_{1}^{+}$can be determined from Eqs. (10) and (13). In the computation program, value of $u_{\infty}^{+}$is allowed to vary for obtaining its critical value which gives best fit to Marušić \& Perry's (1995) experimental data.

The curve giving $u^{+}$as a function of $y^{+}$must have a horizontal tangent at the outer edge of the boundary layer. The value of $y^{+}=y_{o}^{+}$at the outer edge of the boundary layer can not be fixed due to asymptotic behaviour of the curve. The value of non-dimensional velocity $u^{+}=\xi$

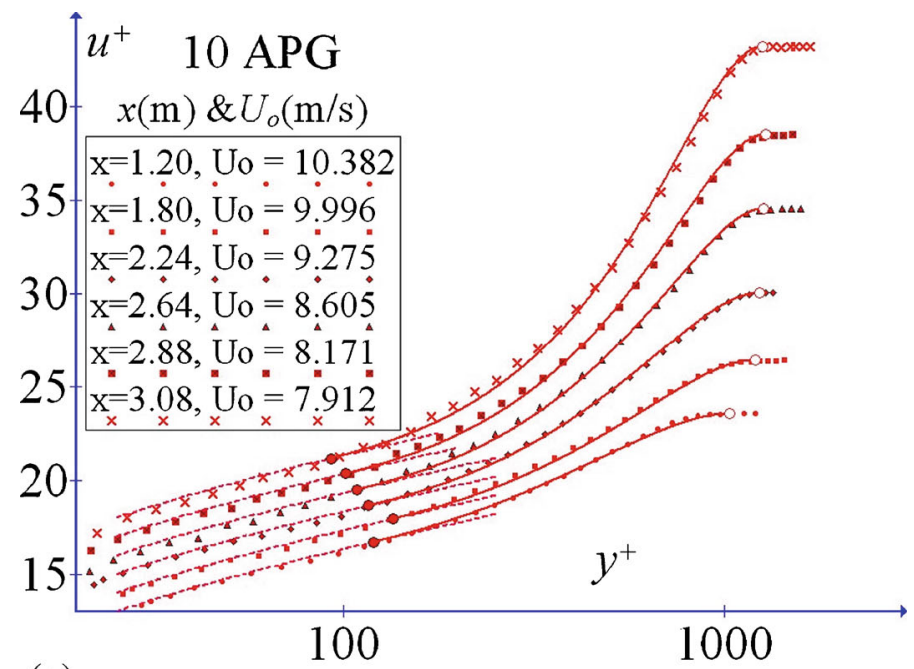

(a)

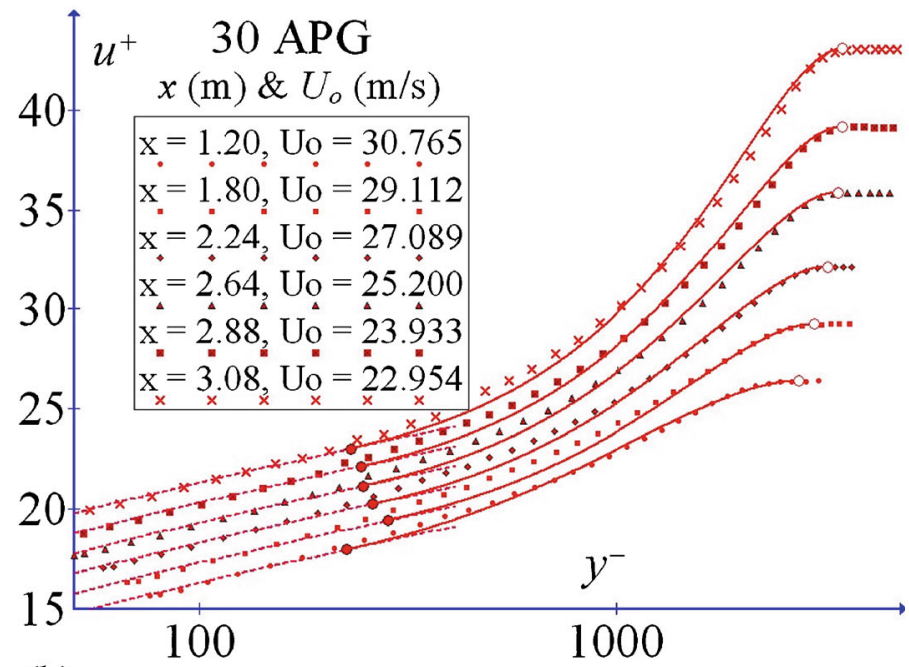

(b)

Figure 6. Law of the wall (dotted line) and law of the wake (bold line) compared with Marušiæ and Perry's data. The points $\left(u_{1}^{+}, y_{1}^{+}\right)$and $\left(\xi, y_{o}^{+}\right)$are shown in closed circle and open circle, respectively. Ordinate scale refers to lowest profile. 
represents, however, the position of the asymptote at the outer edge of the boundary layer. The value of $y_{o}^{+}$at each station may be judged on the basis of few data points around the edge of the boundary layer. Law of the wall $(4,5)$ and law of the wake $(6)$ are compared with measurements of Marušić \& Perry's (1995) in figure 6. In all the cases the fitting is good enough to justify the conclusion that two laws (4 and 5) and (6) are supported by experimental evidence for nonequilibrium turbulent boundary layer with adverse pressure gradient and thus may be used in further deduction.

The values of $u_{1}^{+}, y_{1}^{+}, u_{\infty}^{+}$and other related parameters for 10 and 30 APG flows are given in table 2. The values of the Pearson's correlation coefficients $r$ between two variables (experimental velocity and predicted value) are also given in the last column of the table 2 .

Mazumdar \& Mandal (2009) have shown that locus of $\xi$ for flat plate with zero pressure gradient (ZPG) can be approximated by the following relation

$$
\xi=2.9179626 \ln \left(y_{o}^{+}\right)+3.7427666 .
$$

In figure 7 the relation (14) is drawn along with loci of $\xi$ for 10 and 30 APG flows and loci of $\xi$ of other two adverse pressure gradient flows (Bell 1966; Bradshaw 1967; (data source: Coles $\&$ Hirst 1969) to show how the affect of outside manipulation is taken care of by the quantity $\xi$. One important feature can be noticed that for all the cases of adverse pressure gradient flows, be it in the state of equilibrium or non-equilibrium, the loci of $\xi$ branch off from the locus of $\xi$ for flat plate.

Extent of wall region, $y_{1}^{+}$and the ratio $y_{1}^{+} / y_{o}^{+}$are found to decrease with increase of $R e_{\theta}$ while $y_{o}^{+}$is an increasing function of $R_{\theta}$. In ZPG case both $y_{1}^{+}$and $y_{o}^{+}$are increasing function of Reynolds number but the ratio $y_{1}^{+} / y_{o}^{+}$is decaying function of Reynolds number (Mazumdar \& Mandal 2009). In fact, the ratio $y_{1}^{+} / y_{o}^{+}$is a measure of the non-dimensional quantity $y / \delta$ from the wall below which law of the wall (4) and (5) governs the flow and above which law of the wake (6) takes over. The ratio of $u_{1}^{+} / \xi$ is also decreased with Reynolds number.

Table 2. Values of $y_{o}^{+}, \xi, u_{1}^{+}, y_{1}^{+}, u_{\infty}^{+}, u_{1}^{+} / \xi, y_{1}^{+} / y_{o}^{+}$and $r$.

\begin{tabular}{lccccccccc}
\hline Flow & $\mathrm{x}, \mathrm{m}$ & $y_{o}^{+}$ & $\xi$ & $u_{1}^{+}$ & \multicolumn{1}{c}{$y_{1}^{+}$} & $u_{\infty}^{+}$ & $u_{1}^{+} / \xi$ & $y_{1}^{+} / y_{o}^{+}$ & $\mathrm{r}$ \\
\hline 10 APG & 1.2 & 1029 & 23.56 & 16.71 & 119.9 & 45 & 0.71 & 0.12 & 0.9998 \\
& 1.8 & 1203 & 25.42 & 16.96 & 135.3 & -68 & 0.67 & 0.11 & 0.9995 \\
& 2.2 & 1234 & 28.06 & 16.66 & 116.6 & -20 & 0.59 & 0.09 & 0.9998 \\
& 2.6 & 1265 & 31.54 & 16.50 & 108.4 & -3 & 0.52 & 0.09 & 0.9995 \\
& 2.9 & 1280 & 34.50 & 16.37 & 101.6 & 0 & 0.47 & 0.08 & 0.9996 \\
& 3.1 & 1253 & 38.20 & 16.18 & 92.9 & 2 & 0.42 & 0.07 & 0.9994 \\
APG & 1.2 & 2753 & 26.39 & 17.98 & 224.9 & 37 & 0.68 & 0.08 & 0.9992 \\
& 1.8 & 3003 & 28.23 & 18.44 & 283.7 & -86 & 0.65 & 0.09 & 0.9979 \\
& 2.2 & 3243 & 30.11 & 18.27 & 260.3 & -88 & 0.61 & 0.08 & 0.9988 \\
& 2.6 & 3427 & 32.88 & 18.16 & 246.7 & -25 & 0.55 & 0.07 & 0.9989 \\
& 2.9 & 3503 & 35.15 & 18.15 & 244.5 & -8 & 0.52 & 0.07 & 0.9991 \\
& 3.1 & 3515 & 38.07 & 18.03 & 230.1 & -3 & 0.47 & 0.07 & 0.9993 \\
\hline
\end{tabular}




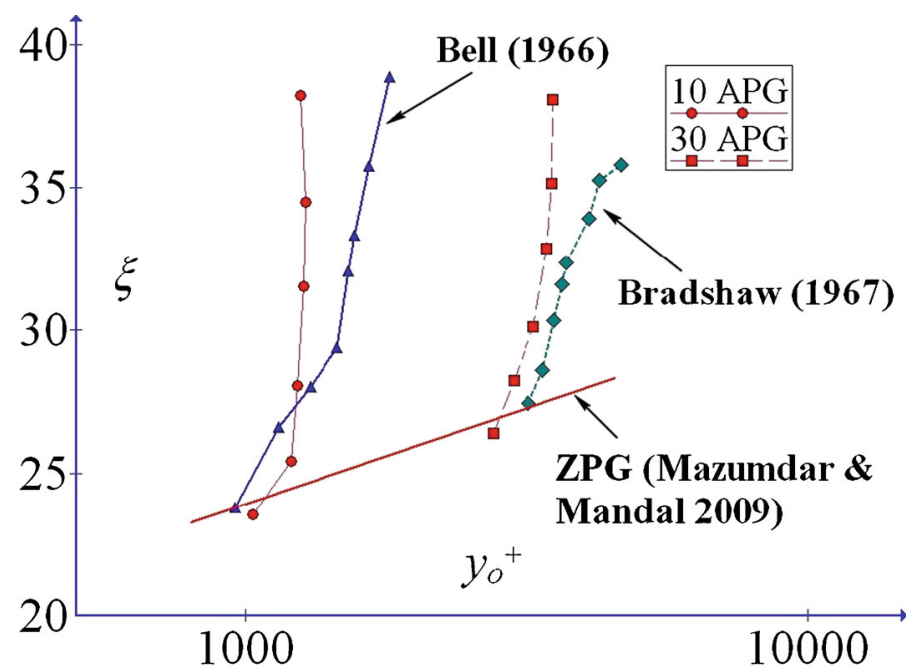

Figure 7. Locus of $\xi$ for ZPG flow compared with APG flows.

\subsection{Wake characteristics}

The Eq. (3) being too simple for describing the wake region of the boundary layer and accordingly based on the idea of Coles (1956) Eq. (3) should be replaced by

$$
u^{+}=f\left(y^{+}\right)+A(x) w(\eta)
$$

where $A(x)$ is the amplitude function, $w(\eta)$ is the wake function and $\eta=y / \delta, \delta$ being the boundary layer thickness.



Figure 8. Relation between maximum values of wake and pressure parameter. 
The wake function is generally defined as the difference between the measured data in the outer region of the boundary layer and an assumed logarithmic law in this region defined by $f\left(y^{+}\right)$. The method to be followed here, for determination of wake, will be somewhat different. Values of wake are determined as difference between the measured data in the outer region of the boundary layer and values obtained from Eqs. (4) and (5) in that region.

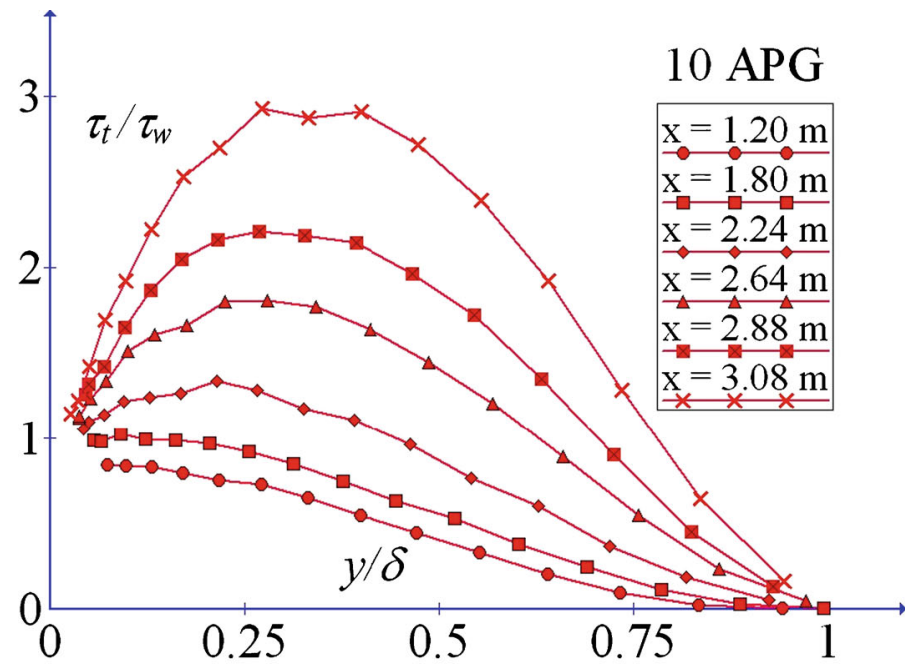

(a)



(b)

Figure 9. Shear stress distribution for 10 and 30 APG flows. 
The maximum value of wake, $w_{\max }$ and its position $\eta_{m}$ at which it occurs, is found by fitting a parabola through three points around the maximum value of wake. Wake function $w(\eta)$ is defined such that

$$
w(\eta)=1 \quad, \quad A(x)=w_{\max }
$$

Here by definition, $w_{\max }$ is somehow related to Coles wake parameter $\Pi$. So, $w_{\max }$ like Coles' wake parameter can be related to the $\beta$. The relation is shown in figure $8 . w_{\max }$ can be approximated by the following relation

$$
w_{\max }=5.644 \beta^{0.5465} .
$$

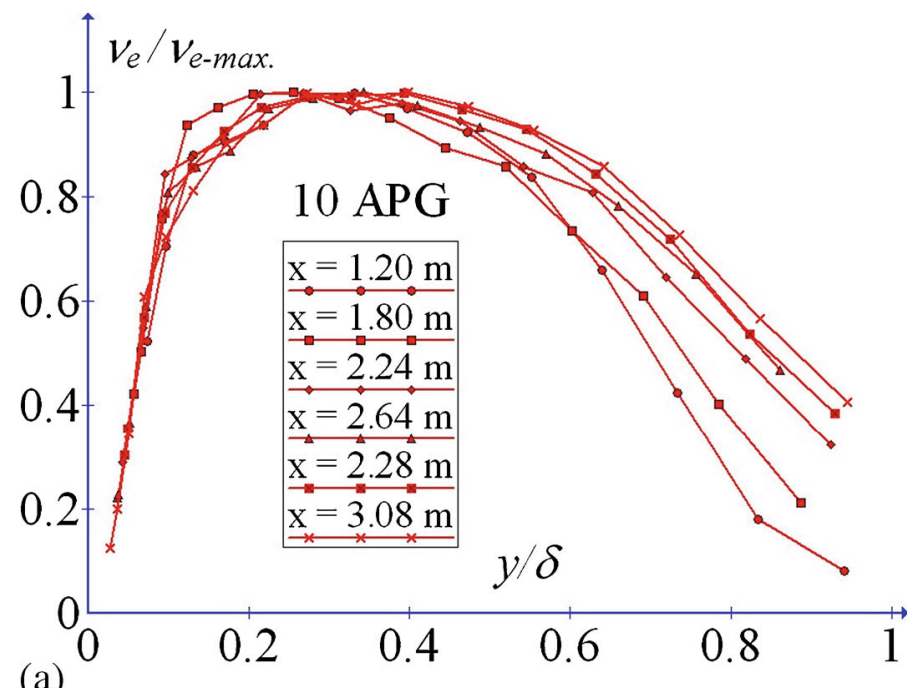

(a)

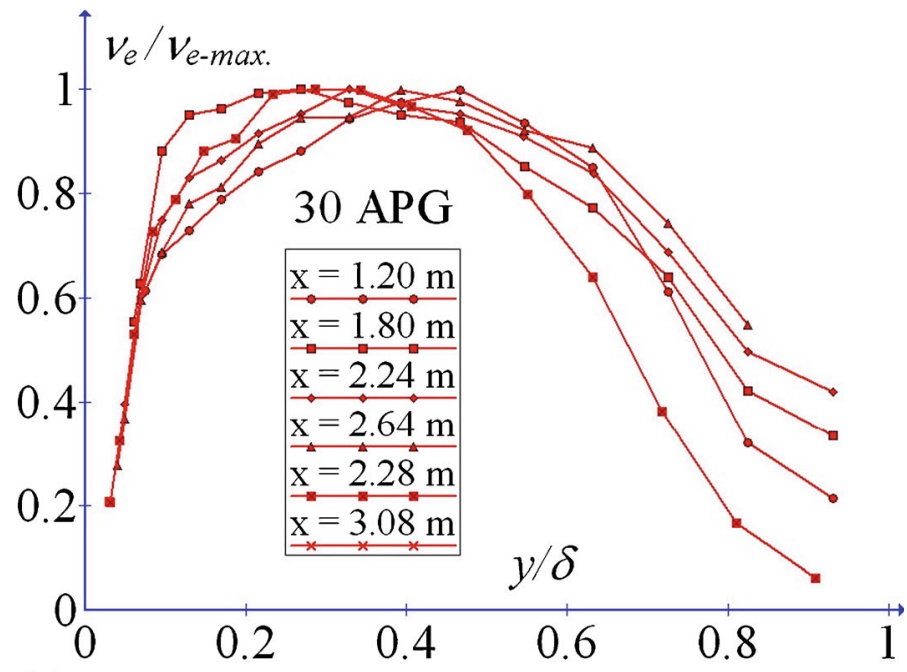

(b)

Figure 10. Eddy viscosity distribution for 10 and 30 APG flows. 


\subsection{Computation of eddy viscosity}

The turbulent shear stress can be described by

$$
\tau_{t}=-\rho \overline{u^{\prime} v^{\prime}}=\mu_{e} \frac{\partial u}{\partial y},
$$

where $\tau_{t}$ is turbulent part of shear stress, $\rho$ is density of fluid, $u^{\prime}$ is stream wise velocity fluctuation, $v^{\prime}$ is vertical velocity fluctuation, $\mu_{e}$ is the physical variable called eddy viscosity (or turbulent viscosity), $u$ is stream wise velocity component (mean), and $y$ is distance from the wall.

From Eq. (18) the eddy viscosity can written as:

$$
\mu_{e}=\frac{\tau_{t}}{\partial u / \partial y}
$$

Dividing by the shear stress at the wall $\tau_{w}$ one obtains:

$$
\frac{\mu_{e}}{\tau_{w}}=\frac{\tau_{t} / \tau_{w}}{\partial u / \partial y},
$$

Or, equivalently

$$
\frac{v_{e}}{v}=\frac{\tau_{t} / \tau_{w}}{d u^{+} / d y^{+}}
$$

where $v_{e}$ is kinematic eddy viscosity $\left(=\mu_{e} / \rho\right)$. This is the general expression for eddy viscosity. With the structure of turbulence already established for two regions, eddy viscosity can be computed, if shear stress distribution is known.

Shear stress distribution for 10 and 30 APG flows are plotted in figure 9. Using figure 9 in association with law of the wall $(4,5)$ and law of the wake (6) and Eq. (20), eddy viscosity is computed and the results are plotted in figure 10.

The diagrams are normalized by the maximum value of the eddy viscosity $\left(v_{e} / v\right)_{\max }$. It reveals from the plot that the points in the outer region of the boundary layer do not gather around a



Figure 11. Values of $\xi$ plotted against maximum eddy viscosity. 
common curve whereas self similarity is observed in the wall region. Position of occurrence of maximum eddy viscosity varies in between $0.1<y / \delta<0.5$.

Correlation between the value of $\xi$ and $\left(v_{e} / v\right)_{\max }$ is shown in figure 11. It is observed that eddy viscosity does depend on $\xi$ as well as the pressure gradient.

\section{Conclusions}

The turbulent boundary layer can be split up into inner region obeyed by the modified Spalding's law and outer region governed by the Persen's wake law. These two laws are quite appropriate for the description of the entire turbulent boundary layer. The common point $\left(u_{1}^{+}, y_{1}^{+}\right)$between the inner and outer region has been determined by employing measured velocity data from both the regions and satisfying a geometrical constraint. Inner region is unaffected by outside manipulation but outer region is affected by outside manipulation which is taken care of by the non-dimensional velocity $\xi$. For flows of same momentum thickness Reynolds number, nondimensional velocity $\xi$ will be higher for the adverse gradient flow than that for the zero pressure gradient flow. Unlike zero pressure gradient flow, extent of near to wall region described by law of the wall i.e., value of $y_{1}^{+}$decreases as Reynolds number increases in an adverse gradient flow. It is observed that mean velocity profiles do not collapse in deficit co-ordinates of Zagarola and Smits. Self similarity in eddy viscosity is preserved in the wall region only. Maximum eddy viscosity not only depends on $\xi$ but also on the pressure gradient.

A remark may finally be made that law of the wall $(4,5)$ and law of the wake $(6)$, as examined above in the light of Marušić \& Perry's (1995) measurements, are capable of explaining the structures of turbulence that prevail throughout the non-equilibrium adverse pressure gradient layer.

\section{Notations}

A Constant appearing in Spalding's formula

$A(x)$ Amplitude function

$C_{p}$ Coefficient of pressure

$P, p$ Pressure

$\operatorname{Re}_{\theta}$ Momentum thickness Reynolds number, $U_{o} \theta / \nu$

$r$ Pearson's correlation coefficient

$u$ Measured velocity

$U_{o}$ Velocity at the edge of the boundary layer/free stream velocity

$U_{\text {ref }}$ Reference velocity

$u^{+}$Non-dimensional velocity, $u v_{*} / v$

$-\rho \frac{u_{\infty}^{+}}{u^{\prime} v^{\prime}}$ Constant appearing in Persen's wake law

$x$ Down stream distance

$y$ Distance from the wall

$y^{+}$Non-dimensional wall distance, $y v_{*} / v$

$y_{o}^{+}$Non-dimensional boundary layer thickness, $\delta v_{*} / v$

$u_{1}^{+}, y_{1}^{+}$Coordinate of the meeting point of wall region and wake region

$w(\eta)$ Wake function

$w_{\max }$ Maximum value of wake 


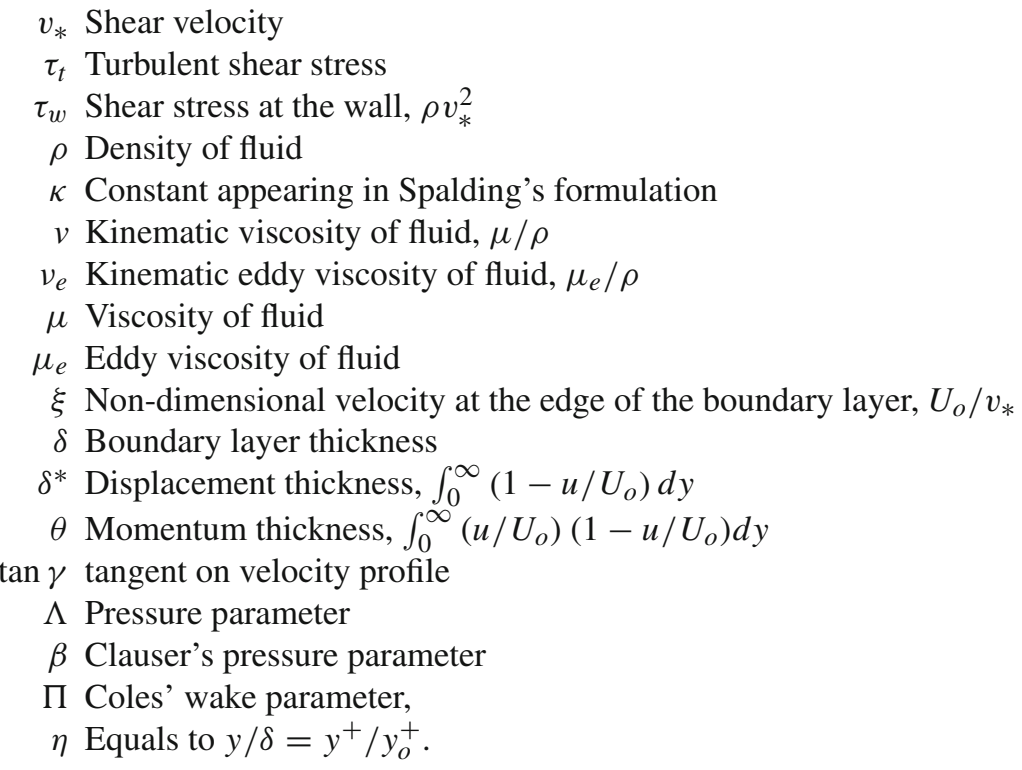

\section{Acknowledgements}

The Authors wish to thank the unknown reviewer for his many pertinent comments and suggestions.

\section{References}

Aubertine C D and Eaton J K 2005 Turbulence development in a non-equilibrium turbulent boundary layer with mild adverse pressure gradient. J. Fluid Mech. 532: 345-364

Bell J 1966 Forced turbulent convective heat transfer from a flat plate in adverse pressure gradients, Mechanical Engineering Thesis University of Melbourne, Australia

Bradshaw P 1967 The response of a constant-pressure turbulent layer to the sudden application of an sudden adverse pressure gradient, National Physics Laboratory Aero Report 1219

Castillo L and George W K 2001 Similarity analysis for turbulent boundary layer with pressure gradient : Outer flow. AIAA J. 39: 41-47

Castillo L and Wang X 2004 Similarity analysis for non-equilibrium turbulent boundary layers. J. Fluids Eng. 126: 827-834

Clauser F H 1954a Turbulent boundary layers in adverse pressure gradients. J. Aero. Sci. 21: 91-108

Clauser F H 1954b The turbulent boundary layer. Adv. Appl. Mech. 4: 1-54

Coles D E 1956 The law of the wake in the turbulent boundary layer. J. Fluid Mech. 1: 191-226

Coles D E and Hirst E A 1969 Proceedings computation of turbulent boundary layer -1968, AFOSR-IFPStanford Conference, Vol. II, Stanford University

Gorin A V and Sikovsky D P 1998 Turbulent boundary layers with strong adverse pressure gradients. $J$. Thermophys. Aeromech. 5: 313-330

Kornilov V I and Litvinenko Y A 2001 Skin friction measurements in an incompressible turbulent boundary layer. part 1. Adverse pressure gradient. J. Thermophys. Aeromech. 8: 475-491

Maciel Y, Rossignol K S and Lemay J 2006 Self-similarity in the outer region of adverse-pressure-gradient turbulent boundary layers. AIAA J. 44: 2450-2464 
Marušić I and Perry A E 1995 A wall-wake model for the turbulence structure of boundary layers. part 2. Further experimental support. J. Fluid Mech. 298: 389-407

Materny M, Drozdz A, Drobniak S and Elsner W 2008 Experimental analysis of turbulent boundary layer under the influence of adverse pressure gradient. Arch. Mech. 60(6): 449-466

Mazumdar H P and Mandal B C 2009 On Persen theory of two dimensional turbulent boundary layer. $J$. Appl. Mech. Eng. 14: 1009-1028

Österlund J M 1999 Experimental studies of zero pressure - gradient turbulent boundary layer flow. Ph. D. Thesis, Royal Institute of Technology, Stockholm, Sweden

Perry A E and Marušić I 1995 A wall-wake model for the turbulence structure of boundary layers. Part 1. Extension of the attached eddy hypothesis. J. Fluid Mech. 298: 361-388

Persen L N 1976 The turbulent boundary layer and the closure problem. Proc. of AGARD Conf. on Turbulent Boundary Layer- Experiments, Theory and Modelling. 271: 17.1-17.15, Den Hagg

Spalding D B 1961 A single formula for the law of the wall. J. Appl. Mech, 28: 455-458

Townsend A A 1976 The Structure of Turbulent Shear Flow, Cambridge University Press, $2^{\text {nd }}$ ed

Zagarola M V and Smits A J 1998 Mean-flow scaling of turbulent pipe flow. J. Fluid Mech. 373: 33-79 